MTC 2005 - Instrumentation and Measurement

Technology Conference

Ottawa, Canada, 17-19 May 2005

\title{
Millimeter Wave Detection of Localized Anomalies in the Space Shuttle External Fuel Tank Insulating Foam and Acreage Heat Tiles
}

\author{
S. Kharkovsky ${ }^{1}$, J.T. Case ${ }^{1}$, R. Zoughi ${ }^{1}$ and F. Hepburn ${ }^{2}$ \\ 'Applied Microwave Nondestructive Testing Laboratory (amntl) \\ Electrical and Computer Engineering Department \\ Universiiy of Míissuu i-Rüliā \\ Rolla, Missouri 65409 \\ Email: zoughir@umr.edu, URL: http://www.ect.umr.edu/amntl \\ ${ }^{2}$ NASA Marshall Space Flight Center \\ Marshall Space Flight Center, AL 35812
}

\begin{abstract}
The Space Shuttle Columbia's catastrophic accident emphasizes the growing need for developing and applying effective, robust and life-cycle oriented nondestructive testing (NDT) methods for inspecting the shuttle external fuel tank spray on foam insulation (SOFI) and its protective acreage heat tiles. Millimeter wave NDT techniques were one of the methods chosen for evaluating their potential for inspecting these structures. Several panels with embedded anomalies (mainly voids) were produced and tested for this purpose. Near-field and far-field millimeter wave NDT methods were used for producing millimeter wave images of the anomalies in SOFI panel and heat tiles. This paper presents the results of an investigation for the purpose of detecting localized anomalies in two SOFI panels and a set of heat tiles. To this end, reflectometers at a relatively wide range of frequencies (Ka-band (26.5 - $40 \mathrm{GHz}$ ) to W-band (75-110 GHz)) and utilizing different types of radiators were employed. The results clearly illustrate the utility of these methods for this purpose.
\end{abstract}

Keywords - millimeter waves, nondestructive testing, near-field, far-field, space shuttle, external tank, insulating foam, heat tiles.

\section{INTRODUCTION}

The Space Shuttle Columbia's catastrophic failure has been attributed to a piece of external tank spray on foam insulation (SOFI) striking the leading edge of the left wing of the orbiter causing significant damage to some of the protecting heat tiles [1]. There is an urgent need for an advance nondestructive testing (NDT) technique capable of inspecting the external tank SOFI during and subsequent to the application of the foam and prior to a launch. It is also extremely desirable, from safety, cost saving, personnel training and a unified inspection approach, to apply the same inspection method to evaluate the integrity of the critical bond between the orbiter's protective (acreage) heat tiles and its fuselage prior and subsequent to a launch. Such a comprehensive inspection technique enables NASA to perform life-cycle inspection on critical components of the orbiter and its supporting hardware. Consequently, NASA Marshall Space Flight Center initiated an investigation into several potentially viable NDT techniques for this purpose [25]. One such method involves the use of millimeter wave NDT methods to achieve these goals [2,5]. This paper presents the results of an investigation for the purpose of detecting localized anomalies in two SOFI panels and a set of heat tiles. To this end, reflectometers at a relatively wide range of frequencies (Ka-band $(26.5-40 \mathrm{GHz})$ to W-band $(75-110 \mathrm{GHz}))$ and utilizing different types of radiators were employed.

\section{PANEL SPECIFICATION AND APPROACH}

Millimeter wave NDT methods have been used in a wide range of applications [6]. The relatively small wavelengths associated with signals at millimeter wave frequencies make them attractive for inspecting low loss dielectric materials. These methods are also capable of producing high spatial resolution images of the interior of various complex, thick and layered composite structures [6,7]. The Space Shuttle's external fuel tank SOFI is in the family of low permittivity and low loss dielectric materials. The relative dielectric properties of the SOFI was measured at X-band, using a completely-filled rectangular waveguide approach, to be $\varepsilon_{r}=$ $1.05-\mathrm{j} 0.003$ [2]. This is expected since the foam is primarily composed of small air bubbles contained in low permittivity and low loss polymers. In addition, the SOFI is a homogeneous material at millimeter wave frequencies due to the small size of the air bubbles compared to the operating wavelengths (i.e., several orders of magnitude smaller). The acreage heat tiles are multi-layered structures composed of a variety of ceramic-like materials capable of insulating the fuselage of the orbiter from extreme heat encountered during its re-entry into the atmosphere. The heat tiles are also in the family of low loss dielectric materials but with higher relative permittivity than the SOFI.

Figure la shows a picture of one of the SOFI panels used in this investigation. This panel (labeled A1) was composed of a $300 \mathrm{~mm}$ by 300 wide and $75 \mathrm{~mm}$ thick SOFI adhered to an aluminum substrate. 


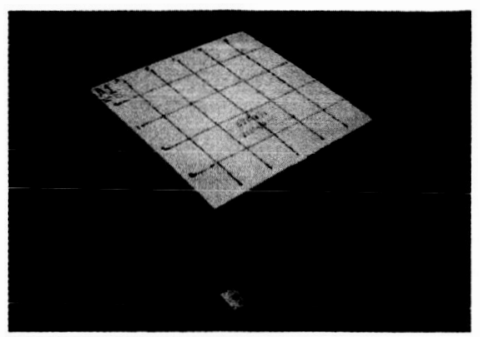

(a)

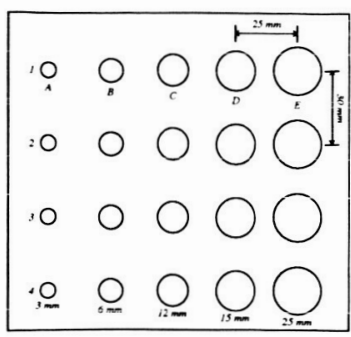

(b)
Figure 1: The $75 \mathrm{~mm}$-thick SOFI panel: (a) picture; (b) top view schematic with twenty embedded voids.

Twenty void-like anomalies were produced in the interior of this panel, ranging in diameter and height from $3 \mathrm{~mm}$ to 25 $\mathrm{mm}$. Consequently, the largest void was $25 \mathrm{~mm}$ in diameter and $25 \mathrm{~mm}$ in height, while the smallest was $3 \mathrm{~mm}$ in diameter and $3 \mathrm{~mm}$ in height. Therefore, some of these anomalies resembled voids while those with larger diameters than height resembled unbonds (or delamination within the SOFI). The top view schematic of this panel is shown in Fig.lb. These voids were placed on the substrate according to the schematic shown in Fig.2a. Another SOFI panel used in the investigation (labeled A5) had the same dimensions and voids as in panel $\mathrm{Al}$, except that the voids were located at different depths within the SOFI thickness, as shown in Fig.2b.

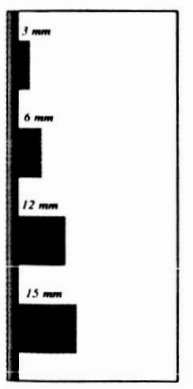

(a)

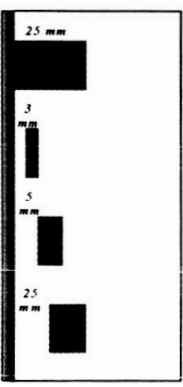

(b)
Figure 2: Side view schematic of $75 \mathrm{~mm}$-thick SOFI panel for $25 \mathrm{~mm}$ diameter voids: (a) panel A1; (b) panel A5.

The acreage heat tile specimen was composed of nine individual square tiles each with an approximate area of 150 $\mathrm{mm}$ by $150 \mathrm{~mm}$ with varying thicknesses in the range of 15 $\mathrm{mm}$ to $30 \mathrm{~mm}$ (even within a given tile) and three rectangular tiles with an approximate area of $75 \mathrm{~mm}$ by $150 \mathrm{~mm}$. Various anomalies representing thin unbonds and repaired regions were embedded in these tiles.

In this investigation, several laboratory designed millimeter wave reflectometers were used for inspecting these panels at relatively wide range of frequencies encompassing Ka-band $(26.5-40 \mathrm{GHz})$ to $\mathrm{W}$-band $(75-110$ $\mathrm{GHz}$ ). Two methods were used for inspecting these panels. One consisted of the reflectometers in conjunction with radiators such as either open-ended rectangular waveguide probes or small horn antennas with the panels primarily placed in the near-field region of these radiators. The second method consisted of inspecting the panels in the far-field of a focused lens antenna (only at $100 \mathrm{GHz}$ ). Open-ended rectangular waveguide radiators are not as efficient as horn antennas. However, when a specimen is placed in the nearfield region of such probes resulting images possess relatively high spatial resolution. This is due to the fact that when operating in the near-field region of a probe spatial resolution is primarily a function of the probe size [6]. Horn antennas on the other hand are relatively efficient radiators. Lens antennas produce a reasonably small footprint in their farfield (far-field focusing) [8].

The SOFI and the heat tile panels were then placed on 2D automated scanning tables. The reflectometers were held at a fixed position above the panels while the table moved the panels in a 2D raster format. In this way a 2D scan/image of the panels was produced at different frequencies and standoff distances (e.g., the distance between the radiator and the surface of a panel). A dc voltage, proportional to the reflected signal characteristics (i.e., phase or magnitude) from the panel under test was then measured and recorded in a matrix corresponding to the scanning area. Subsequently, the measured voltages in this matrix were normalized (with respect to the highest voltage value) and a gray-scale image of the panel was produced.

\section{RESULTS}

\section{A. SOFI Panels}

Figures 3 shows the images of the SOFI panel A1 obtained at different frequencies using the near-field approach with a small horn antenna (Figs.3a -3c) as wel! as the far-field approach with a focused lens antenna (Fig.3d). In Fig.3a the standoff distance for the Ka-band horn was $15 \mathrm{~mm}$. The voids are indicated by circular features in the image. The indication at the bottom right hand corner of the image is that from a void with a diameter of $25 \mathrm{~mm}$ and a height of $15 \mathrm{~mm}$ placed on the aluminum substrate, while the indication at the top right hand corner is due to a similar void but with a height of $3 \mathrm{~mm}$. As expected, the former indication is stronger than the latter due to the difference between their respective heights. On the other hand, it was unexpected to see that the indications of voids located in the middle of the panel, along rows 2 and 3, are stronger than indications of larger voids, as will be explained later. From Fig.3a it is clear that at least seventeen of the voids are readily detected. The three voids that are not readily detected (the three voids from the top in the left hand column) correspond to the smallest voids in this panel with diameters of $3 \mathrm{~mm}$ and heights of $3 \mathrm{~mm}, 6 \mathrm{~mm}$ and $12 \mathrm{~mm}$, respectively. However, some of these voids were detected at higher frequencies. For instance, the smallest void with diameter of $3 \mathrm{~mm}$ and height of $3 \mathrm{~mm}$ can be seen in the top right corner of the $70 \mathrm{GHz}$ image of the panel shown in 
Fig.3b (obtained with a small horn antenna at the standoff distance of $5 \mathrm{~mm}$ ). This illustrates the higher spatial resolution at $70 \mathrm{GHz}$. Moreover, higher frequency reflectometers provide detection of natural voids in the SOFI panel and anomalies (non-uniformities) in the adhesive layer between SOFI and the substrate (Figs.3b-3d).

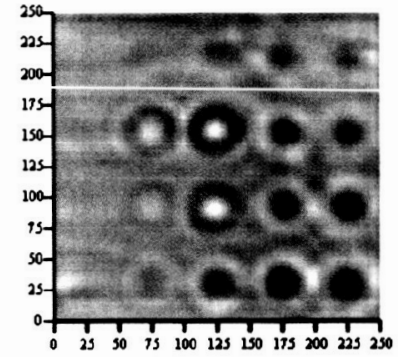

(a)

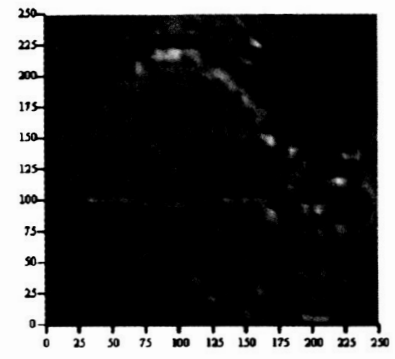

(c)

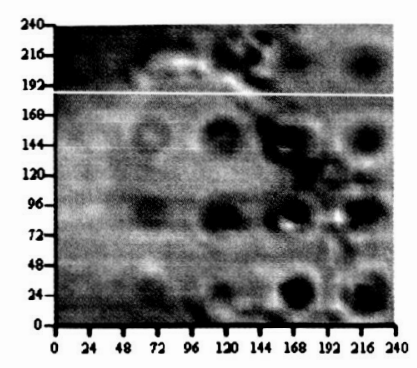

(b)

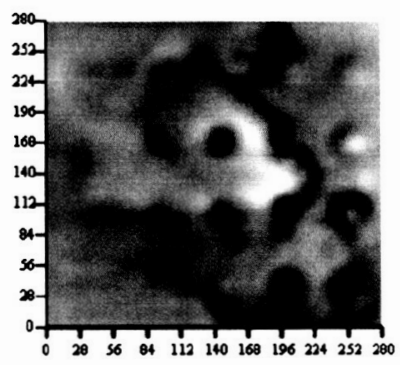

(d)
Figure 3: Millimeter wave image of panel $\mathrm{Al}$ at different frequencies (dimensions are in $\mathrm{mm}$ ): (a) $\mathrm{f}=33.5 \mathrm{GHz}$ with a horn antenna; (b) $\mathrm{f}=70$ $\mathrm{GHz}$ with a horn antenna; (c) $\mathrm{f}=100 \mathrm{GHz}$ with a horn antenna; (d) $\mathrm{f}=100$ $\mathrm{GHz}$ with a lens antenna.

The $100 \mathrm{GHz}$ image in Fig. 3c, using a smail horn antenna, provides more details about the adhesive layer than the other images, as expected. Figure 3d shows the image of the panel at $100 \mathrm{GHz}$ using a focused lens antenna. The voids are indicated in Fig.3d as dark and bright spots with dimensions that are close to their actual dimensions. However, small voids in this panel were not detected with the lens antenna either, because of its high sensitivity to non-uniformities of the adhesive layer which masked these small voids. Distortions associated with the non-uniformities of the adhesive layer are visible as curved shadows and inside the voids with diameter of $25 \mathrm{~mm}$ (right hand column) due to the fact that the thickness of the adhesive layer gradually varied. From Figs.3b-3d it is clear that the mentioned above unexpected strong indication of voids located in the middle of the panel at Ka-band (see Fig.3a) are due to the fact that they are located on top of the thicker adhesive portions. The SOFI panel A5 was scanned using small horn antennas at Ka-band and V-band, as shown in Fig.4.

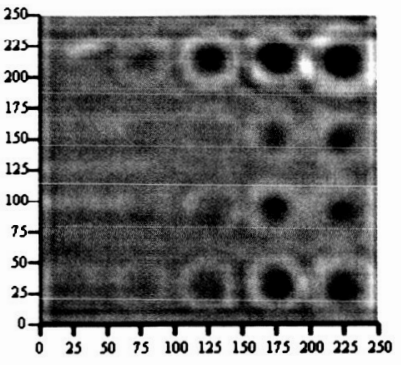

(a)

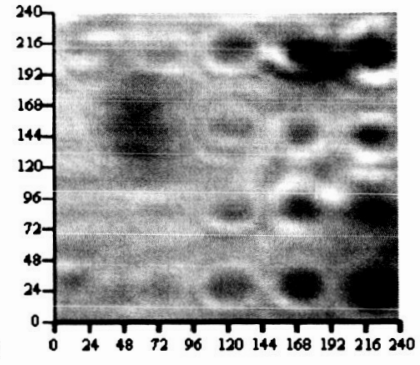

(b)
Figure 4: Millimeter wave image of SOFI panel A5 at different frequencies using a small horn antenna (dimensions are in $\mathrm{mm}$ ): (a) $f=33.5 \mathrm{GHz}$; (b) $\mathrm{f}=$ $70 \mathrm{GHz}$.

In both images the voids are indicated by circular features similar to the image of panel Al. The indication at the top right hand corner of the image is that from a void with a diameter of $25 \mathrm{~mm}$ and a height of $25 \mathrm{~mm}$ placed on the aluminum substrate, while the indication at the bottom right hand corner is due to the void with a diameter of $25 \mathrm{~mm}$ and a height of $12 \mathrm{~mm}$ located at a depth of $13 \mathrm{~mm}$ (see Fig.2b). As expected, the former indication is much stronger than the latter due to the difference between their respective heights and locations. The three voids that are not visible in Fig.4 (the three voids from the bottom in the left hand column) correspond to the smallest voids with diameters of $3 \mathrm{~mm}$ and heights of $12 \mathrm{~mm}, 6 \mathrm{~mm}$ and $3 \mathrm{~mm}$, respectively. These three voids were placed $12 \mathrm{~mm}, 6 \mathrm{~mm}$ and $3 \mathrm{~mm}$ above the aluminum substrate, respectively. It should be noted that these voids could be masked by the non-uniformity associated with the adhesive layer which can be seen near their locations in the V-band image (a dark area in Fig. 4b). Comparison between the $70 \mathrm{GHz}$ images of panels $\mathrm{Al}$ and $\Lambda 5$ (Figs. $3 \mathrm{~b}$ and $4 \mathrm{~b}$ ) shows that the adhesive layer in panel A5 was more uniform than the adhesive layer in panel Al. Nevertheless, the image of panel A5 indicates nonuniformities of the adhesive in the top and bottom right corners of the panel as well as in the area of the two left hand columns in the middle of the panel. The SOFI in panel A5 was cut away at the base from the substrate and subsequently placed on a different aluminum substrate without using any adhesive. Figure 5 shows the image of this panel at $70 \mathrm{GHz}$ indicating the absence of the adhesive layer and its nonuniformity.

It must be noted that Figs.3-5 are raw images and no signal/image processing was applied to them. This is significant since it shows the effectiveness of these millimeter wave NDT methods for producing rapid and informative images about the interior of SOFI. The results shown in Figs.3-5 also indicate that these raw images can provide reasonably close estimate of the void diameters. Clearly, the images are a combination of probe radiation patterns and void geometries and dimensions. Therefore, it is possible to use a deconvolution algorithm to remove the effect of probe radiation pattern from these images resulting a closer size 
estimate and shape of a void [9, 10]. The results also show the ability to closely determine the relative location of a void in an extended SOFI panel.

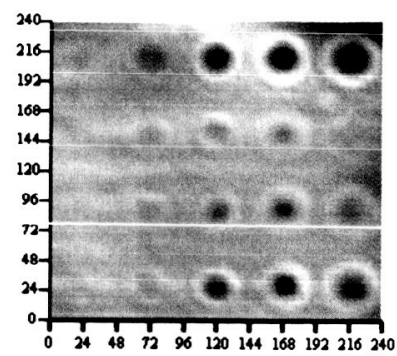

Figure 5: $70 \mathrm{GHz}$ image of SOFI panel A5 (dimensions are in $\mathrm{mm}$ ) without adhesive between foam and a metal substrate.

\section{B. Heat Tiles}

Figure 6 shows the image of the heat tile panel at a frequency of $30 \mathrm{GHz}$ using an open-ended rectangular waveguide probe. The anomalies in five of the tiles are clearly evident.

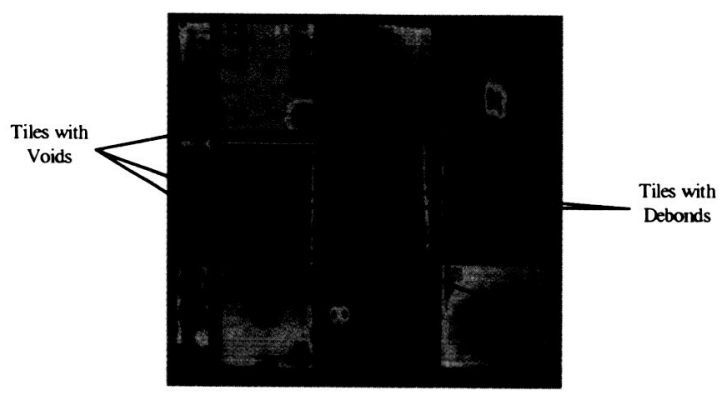

Figure 6: limage of heat tile panel at a frequency of $30 \mathrm{GHz}$ with open ended waveguide probe.

The images of the three square tiles possessing unbond (i.e., diagonal in the case of the middle square tile) clearly show the unbonds and their respective spatial extents. The repair rigions in the two tiles are also detected. The image in Fig. 6 serves as a clear indication of the potential capabilities of these NDT methods for inspecting the acreage heat tiles. It is important to note that the reflectometer used for this purpose is similar to that used for the SOFI inspection. As mentioned in the outset, this is an important issue from the unified inspection point of view (e.g., reflectometer systems capable of inspecting the SOFI and the acreage heat tiles). This panel is currently being scanned at other frequencies, the results of which will be reported in the final extended paper.

\section{SUMMARY}

Undesired manufactured or in-service produced anomalies in the form of voids in the SOFI and unbonds in the heat tiles can significantly reduce their designed effectiveness.
Additionally, unbonds in the heat tiles may cause their complete separation from the fuselage exposing it to extreme heat. Millimeter wave NDT methods are viable candidate for life-cycle inspection of the SOFI and the acreage heat tiles of the Space Shuttle fleet. The results of these investigations described in this paper clearly showed the utility of these methods for detecting localized anomalies in thick SOFI and acreage heat tiles. These methods are fast and robust and the systems used to produce these images are small, real-time and provide a significant amount of useful information about the nature of an anomaly (e.g., size, location, etc.) without the need for complex image processing. This investigation is currently ongoing and additional results will be provided in the final paper.

Acknowledgment: Funding for this work was provided by the NASA Marshall Space Flight Center through a Cooperative Agreement.

\section{REFERENCES}

[1] Columbia Accident Investigation Board Report, NASA, August 2003.

[2] Shrestha S., S. Kharkovsky, R. Zoughi, F.L. Hepburn and G. Workman, "Microwave Nondestructive Inspection of Thick Insulating Foam," The American Society for Non-Destructive Testing (ASNT) Fall Conference and Quality Testing Show, Pittsburgh, PA, 13-17 October 2003

[3] Davis C., F. Santos, "Shearography NDE of Space Launch Vehicles," The American Society for Non-Destructive Testing (ASNT) Fall Conference and Quality Testing Show, Pittsburgh, PA, 13-17 October 2003.

[4] Madaras E., "Terahertz NDE for Inspection of Shuttle Foam," The American Society for Non-Destructive Testing (ASNT) Fall Conference and Quality Testing Show, Pittsburgh, PA, 13-17 October 2003.

[5] Shrestha S., S. Kharkovsky, R. Zoughi, and F.L. Hepburn, "Microwave and Millimeter Wave Nondestructive Evaluation of the External Tank insulating Foam," Materials Evaluation, vol.63, N3, pp.339-344, March 2005.

[6] Zoughi, R., Microwave Non-Destructive Testing and Evaluation, Kluwer Academic Publishers, The Netherlands, 2000.

[7] Bakhtiari, S., S. Ganchev, N. Qaddoumi and R. Zoughi, "Microwave Non-Contact Examination of Disbond and Thickness Variation in Stratified Composite Media," IEEE Transactions on Microwave Theory and Techniques, vol. 42, no. 3, pp. 389-395, March, 1994.

[8] Balanis, C.A., Antenna Theory: Analysis and Design, 2nd Edition, John Wiley and Sons, NY, NY, 1997.

[9] Nandi, A.K. and D. Mampel, "Deconvolution of Ultrasonic Signals in Nondestructive Testing Applications," Proceedings of IEEE Signal Processing/ATHOS Workshop on Higher-Statistics, Girona, Spain, pp. 243-247, June 1995.

[10] Hayward, G. and J.E. Lewis, "Comparison of Some Non-Adaptive Deconvolution Techniques for Resolution Enhancement of Ultrasonic Data", Ultrasonics, vol. 27, no. 5, pp. 155-164, 1977. 